OPEN ACCESS

Edited by:

Peter E. Larsen,

Argonne National Laboratory (DOE),

United States

Reviewed by:

Yuzhen Ye,

Indiana University Bloomington,

United States

Robin Anderson,

Agricultural Research Service (USDA),

United States

*Correspondence:

Laurence G. Rahme

rahme@molbio.mgh.harvard.edu

Specialty section:

This article was submitted to

Systems Microbiology,

a section of the journal

Frontiers in Microbiology

Received: 04 March 2017

Accepted: 14 June 2017

Published: 30 June 2017

Citation:

Bandyopadhaya A, Tsurumi A and

Rahme LG (2017) NF- $\kappa$ Bp50

and HDAC1 Interaction Is Implicated

in the Host Tolerance to Infection

Mediated by the Bacterial Quorum

Sensing Signal

2-Aminoacetophenone.

Front. Microbiol. 8:1211.

doi: 10.3389/fmicb.2017.01211

\section{NF- $\kappa$ Bp50 and HDAC1 Interaction Is Implicated in the Host Tolerance to Infection Mediated by the Bacterial Quorum Sensing Signal 2-Aminoacetophenone}

\author{
Arunava Bandyopadhaya ${ }^{1,2,3}$, Amy Tsurumi ${ }^{1,2,3}$ and Laurence G. Rahme ${ }^{1,2,3 *}$ \\ ${ }^{1}$ Department of Surgery, Massachusetts General Hospital and Harvard Medical School, Boston, MA, United States, \\ ${ }^{2}$ Department of Microbiology and Immunobiology, Harvard Medical School, Boston, MA, United States, ${ }^{3}$ Shriners Hospitals \\ for Children Boston, Boston, MA, United States
}

Some bacterial quorum sensing (QS) small molecules are important mediators of inter-kingdom signaling and impact host immunity. The QS regulated small volatile molecule 2-aminoacetophenone (2-AA), which has been proposed as a biomarker of Pseudomonas aeruginosa colonization in chronically infected human tissues, is critically involved in "host tolerance training" that involves a distinct molecular mechanism of host chromatin regulation through histone deacetylase (HDAC)1. 2-AA's epigenetic reprogramming action enables host tolerance to high bacterial burden and permits long-term presence of $P$. aeruginosa without compromising host survival. Here, to further elucidate the molecular mechanisms of 2-AA-mediated host tolerance/resilience we investigated the connection between histone acetylation status and nuclear factor (NF)-кB signaling components that together coordinate 2-AA-mediated control of transcriptional activity. We found increased NF-кBp65 acetylation levels in 2AA stimulated cells that are preceded by association of CBP/p300 and increased histone acetyltransferase activity. In contrast, in 2-AA-tolerized cells the protein-protein interaction between p65 and CBP/p300 is disrupted and conversely, the interaction between p50 and co-repressor HDAC1 is enhanced, leading to repression of the proinflammatory response. These results highlight how a bacterial QS signaling molecule can establish a link between intracellular signaling and epigenetic reprogramming of pro-inflammatory mediators that may contribute to host tolerance training. These new insights might contribute to the development of novel therapeutic interventions against bacterial infections.

Keywords: quorum sensing, 2-aminoacetophenone, inflammatory cytokines, NF-кB, CBP/p300, HDAC1

\section{INTRODUCTION}

Bacterial quorum sensing (QS) systems regulate the expression of multiple virulence factors via small excreted signaling molecules that play important roles in bacterial cell-to-cell communication and inter-kingdom interaction that favor infection (Ng and Bassler, 2009; Parker and Sperandio, 2009; Rutherford and Bassler, 2012; Starkey et al., 2014; Maura et al., 2016). The QS systems 
of the ESKAPE pathogen Pseudomonas aeruginosa are the most well studied systems and shown to be required for acute and chronic infections (Kerr and Snelling, 2009; Gellatly and Hancock, 2013). P. aeruginosa regulates many of its virulence functions via the QS systems, LasR, RhlR, and MvfR (pqsR; Jimenez et al., 2012). LasR and RhlR rely on the signaling molecules N-acyl-homoserine lactones (AHLs; Jimenez et al., 2012), while the quinolone-dependent QS system, MvfR (PqsR) relies on the 4-hydroxy-2-alkylquinolines (HAQs) signaling molecules, 2-heptyl-3,4-dihydroxyquinoline (PQS, Pseudomonas Quinolone Signal), and HHQ (4-hydroxy-2-heptylquinoline) (Deziel et al., 2005; Diggle et al., 2006; Xiao et al., 2006; Williams and Camara, 2009; Drees and Fetzner, 2015; Drees et al., 2016). Apart from their role as QS signal molecules, AHLs and HAQs also modulate immune responses, promote apoptosis, and control chemotaxis, cell proliferation and phagocytosis by regulating host intracellular signaling pathways (Kravchenko et al., 2008; Rumbaugh and Kaufmann, 2012; Holm and Vikstrom, 2014).

Recently, we have reported that MvfR in addition to HAQs also controls the synthesis of the non-HAQ molecule 2-aminoacetophenone (2-AA; Kesarwani et al., 2011; Bandyopadhaya et al., 2012; Que et al., 2013), which is abundantly produced in $P$. aeruginosa-infected human tissues (Cox and Parker, 1979; Scott-Thomas et al., 2010). 2-AA modulates both bacterial and host functions (Kesarwani et al., 2011; Bandyopadhaya et al., 2012; Que et al., 2013). In the bacterium, 2-AA silences the acute virulence functions of the MvfR QS system (Kesarwani et al., 2011) by binding and inhibiting the enzymatic activity of PqsBC (Drees et al., 2016) and promotes antibiotic tolerance at least by interfering with the bacterial translation apparatus (Que et al., 2013). In the host, it acts as an inter-kingdom immunomodulatory signal (Bandyopadhaya et al., 2012) and promotes metabolic changes (Tzika et al., 2013; Bandyopadhaya et al., 2016a). It dramatically reduces $P$. aeruginosa induced mortality by limiting pathogeninduced inflammation and tissue damage (Bandyopadhaya et al., 2012), while enables $P$. aeruginosa to persist at a high burden level (Bandyopadhaya et al., 2012). Its impact on host metabolism may also favor chronic infection (Tzika et al., 2013; Bandyopadhaya et al., 2016a). More recently, we have uncovered that 2-AA acts as a critical mediator (training agent) of host tolerance/resilience $(\mathrm{HT} / \mathrm{R})$ against $P$. aeruginosa through a distinct molecular mechanism of host chromatin regulation that involves histone deacetylases (HDAC) 1 expression and activity (Bandyopadhaya et al., 2016b). HT/R to infections is defined as the host's ability to limit pathogen triggered damage, while permitting pathogen persistence (Raberg, 2014; Richardson, 2016; Meunier et al., 2017; Soares et al., 2017). The host copes with a pathogenic encounter without a reduction in fitness (Ayres and Schneider, 2012; Medzhitov et al., 2012; Soares et al., 2014) and avoids harmful inflammatory responses that can occur during immune-driven resistance (Schmid-Hempel, 2009). Our understanding of the biological mechanisms mediating mutual pathogen-host adaptation and the causes and consequences of variation in HT/R is extremely limited.
Innate immune mechanisms relay on the recognition of conserved patterns of pathogens, through pattern recognition receptors that activate intracellular signaling pathways ultimately regulating the expression of pro-inflammatory mediators, which together coordinate the early host response to infection, that is required for the activation of adaptive immunity (Mogensen, 2009). The magnitude and duration of the inflammatory response is tightly regulated by endogenous host signaling molecules in order to avoid the self-damaging immunopathology that results from an uncontrolled inflammatory response (Iwasaki and Medzhitov, 2004; Newton and Dixit, 2012). On the other hand, pathogens respond to the threat imposed by the immune system by adopting a series of strategies that aim at escaping or reducing the effectiveness of the host defense mechanisms by interfering with molecules involved in inflammatory signaling, eventually promoting long-standing association with host and persistent infections (Ruby and Monack, 2011; Reddick and Alto, 2014). However, the biological mechanisms by which pathogens act upon hosts to persist and support their survival remains largely elusive.

The intracellular host milieu is hostile for bacterial cells because of the host dynamic defense system, which is primarily controlled by the innate effector cells. The nuclear factor (NF)$\kappa \mathrm{B}$ signaling has an essential role in inflammation and innate immunity (Bhatt and Ghosh, 2014). The prototypical NF-кB is a heterodimer consisting of two subunits, p65 and p50. The p65 has the strongest transcriptional activity, whereas the p50 subunit lacks transactivation domain and accounts for the strong DNA-binding affinity (Gilmore, 2006; Sharma et al., 2015). We have reported that the degradation and dissociation of $\mathrm{I} \kappa-\mathrm{B} \alpha$ from NF- $\kappa \mathrm{B}$, the NF- $\kappa \mathrm{Bp} 65$ translocation to the nucleus and binding to the DNA are impaired during 2-AA-mediated tolerance (Bandyopadhaya et al., 2012; Figure 1).

$\mathrm{NF}-\kappa \mathrm{B}$ can influence transcription by recruitment of co-activators such as the acetyltransferase CREB (c-AMP response element binding) binding protein (CBP)/p300 (Vo and Goodman, 2001). The histone acetyltransferase (HAT) domain of $\mathrm{CBP} / \mathrm{p} 300$ modulates transcription by catalyzing the acetylation of promoter-bound histones (Yuan et al., 2013). In addition, HATs also regulate gene expression through acetylation of $\mathrm{NF}-\kappa \mathrm{B}$ p65, which subsequently determines their nuclear interactions with DNA and other transcriptional regulators (Yang et al., 2010). This acetylation is balanced by the removal of acetyl groups by HDACs, which are associated with chromatin compaction and transcriptional repression (Delcuve et al., 2012). Bacteria can impede the dynamic regulation of HATs and HDACs which may affect host innate immunity (Hamon and Cossart, 2008; Bierne et al., 2012).

Here we investigate 2-AAs' regulation on host intracellular signaling and the effects of this regulation in 2-AA-mediated immunomodulation. Our results provide additional new insights into the 2-AA-mediated mechanism of tolerization that might contribute to therapeutic intervention strategies against bacterial infections. 


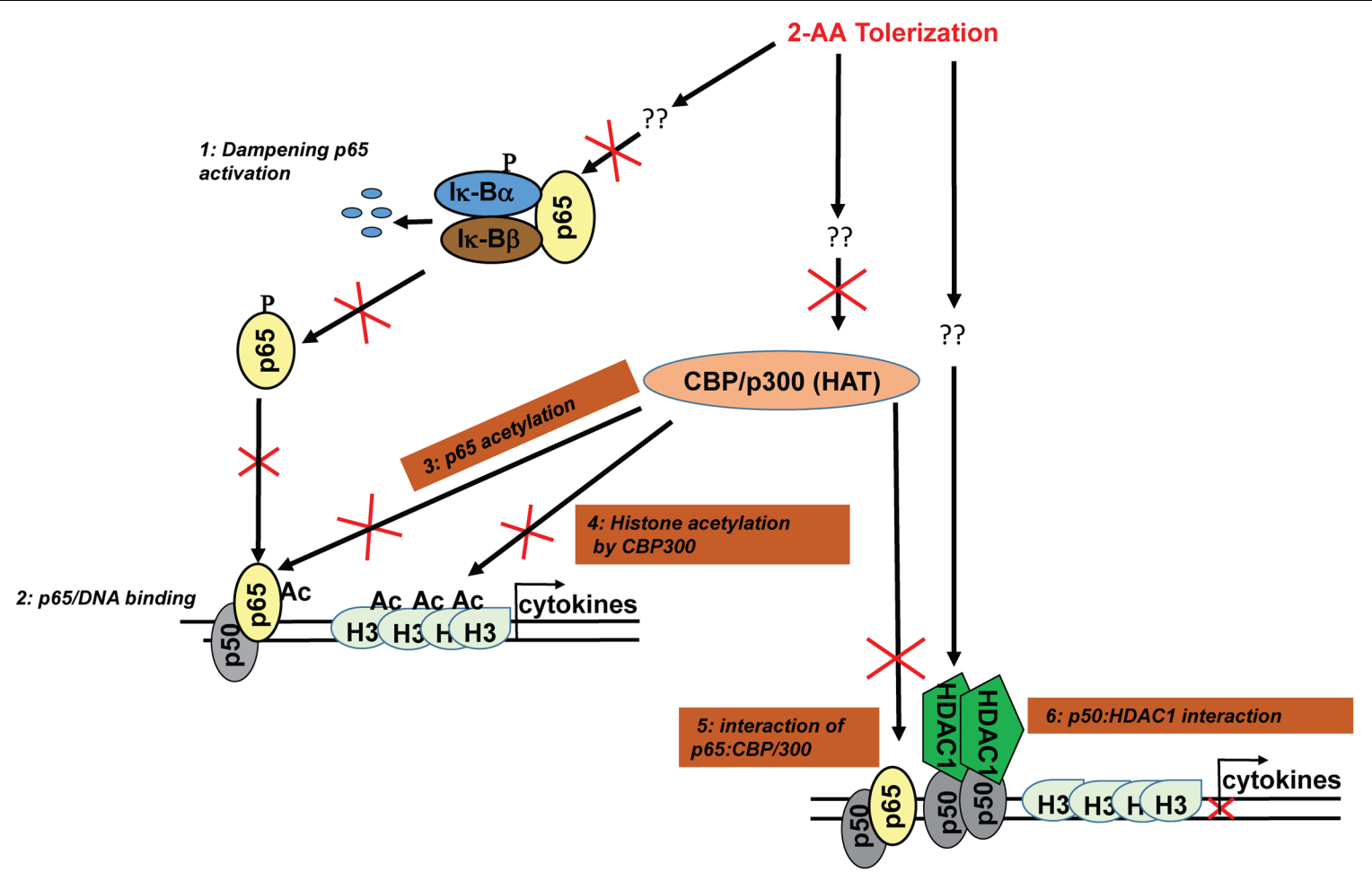

FIGURE 1 | 2-AA-mediated immunomodulation. Our studies have shown that 2-AA tolerization dampens the activation of NF-KB pathway (1) (Bandyopadhaya et al., 2012). The DNA binding activity of NF-кB p65 (2) (Bandyopadhaya et al., 2012) is impaired due to reduced acetylation level of p65 in 2-AA-tolerized cells (3 this study). 2-AA tolerization reduces histone acetylation (H3) via downregulation of CBP/p300 HAT activity and expression (4 this study) and the protein-protein interaction between p65 and CBP/p300 (5 this study). The upregulation of HDAC1 initiates HDAC1-p50 interaction and p50 homodimer-HDAC1 complexes are recruited at targeted sites (6 this study). Consequently, HDAC1 acts to deacetylate $\mathrm{H} 3$ and dampens transcription of cytokines.

\section{MATERIALS AND METHODS}

\section{Cell Culture}

THP-1 Blue cells (human monocytic leukemia line with an NF-кB-inducible reporter; Invivogen, United States) and RAW264.7 cells carrying the NF-кB luciferase plasmid (IMGENEX, United States) were maintained in RPMI 1640 medium (Life Technologies, United States) and Iscove's modified Dulbecco's medium (IMDM, Gibco), respectively. All media was supplemented with 10\% heat-inactivated FBS/1\% AntibioticAntimycotic/2 mM L-glutamine/10 mM HEPES (all from Gibco, United States). The cells were cultured in T-75 flasks (Falcon, United States) and used between passages 2 and 3. HDAC1 knock down and vector control stable cell lines were maintained in complete IMDM (Gibco) with puromycin (Bandyopadhaya et al., 2016b). Cells were maintained in a humidified incubator at $37^{\circ} \mathrm{C}$ in $5 \% \mathrm{CO}_{2}$.

\section{2-AA Tolerization Assay}

THP-1 cells $\left(10^{5} / \mathrm{mL}\right)$ were plated in 24 -well plates. Cells in the treatment groups were pretreated with $400 \mu \mathrm{M}$ 2-AA (SigmaAldrich, United States) for $24 \mathrm{~h}$; pretreated and non-pretreated cells were washed with $1 \times$ phosphate-buffered saline (PBS) and kept in fresh medium (Figure 2A). Cells were stimulated with $200 \mu \mathrm{M} 2$-AA for the durations indicated in the figures. Similarly, murine macrophage RAW264.7 cells were pretreated (or not) with $800 \mu \mathrm{M} 2$-AA for $48 \mathrm{~h}$, then stimulated with $400 \mu \mathrm{M}$ 2-AA.

\section{RNA Isolation and Quantitative RT-PCR}

Total RNA was isolated from approximately $1.2 \times 10^{6}$ cells with the RNeasy Mini Kit (Qiagen, United States) and cDNA was prepared with the RETROscript ${ }^{\mathrm{TM}}$ Kit (Ambion ${ }^{\circledR}$ Life Technologies, United States), as per the manufacturer's instruction. Real-time PCR was conducted using the Brilliant II SYBR green super mix (Agilent, United States) and primer sets for human tumor necrosis factor (TNF; forward: AACATCCAACCTTCCCAAACG, reverse: CTCTTAAACC CCCGAATCCCAG) and GAPDH (forward: CAACAGCGACA CCCACTCCT, reverse: CACCCTGTTGCTGTAGCCAAA). Expression of cytokines was normalized to GAPDH with the $\triangle \mathrm{Ct}$ method and relative expression was calculated relative to non-pretreated and unstimulated control cells. The assay was conducted in triplicate; means and standard deviations were calculated for each group.

\section{Western Blot}

Whole cell lysates were prepared in RIPA buffer (Cell Signaling Technology, United States) supplemented with protease inhibitor cocktail (Sigma). The concentration of protein was determined 


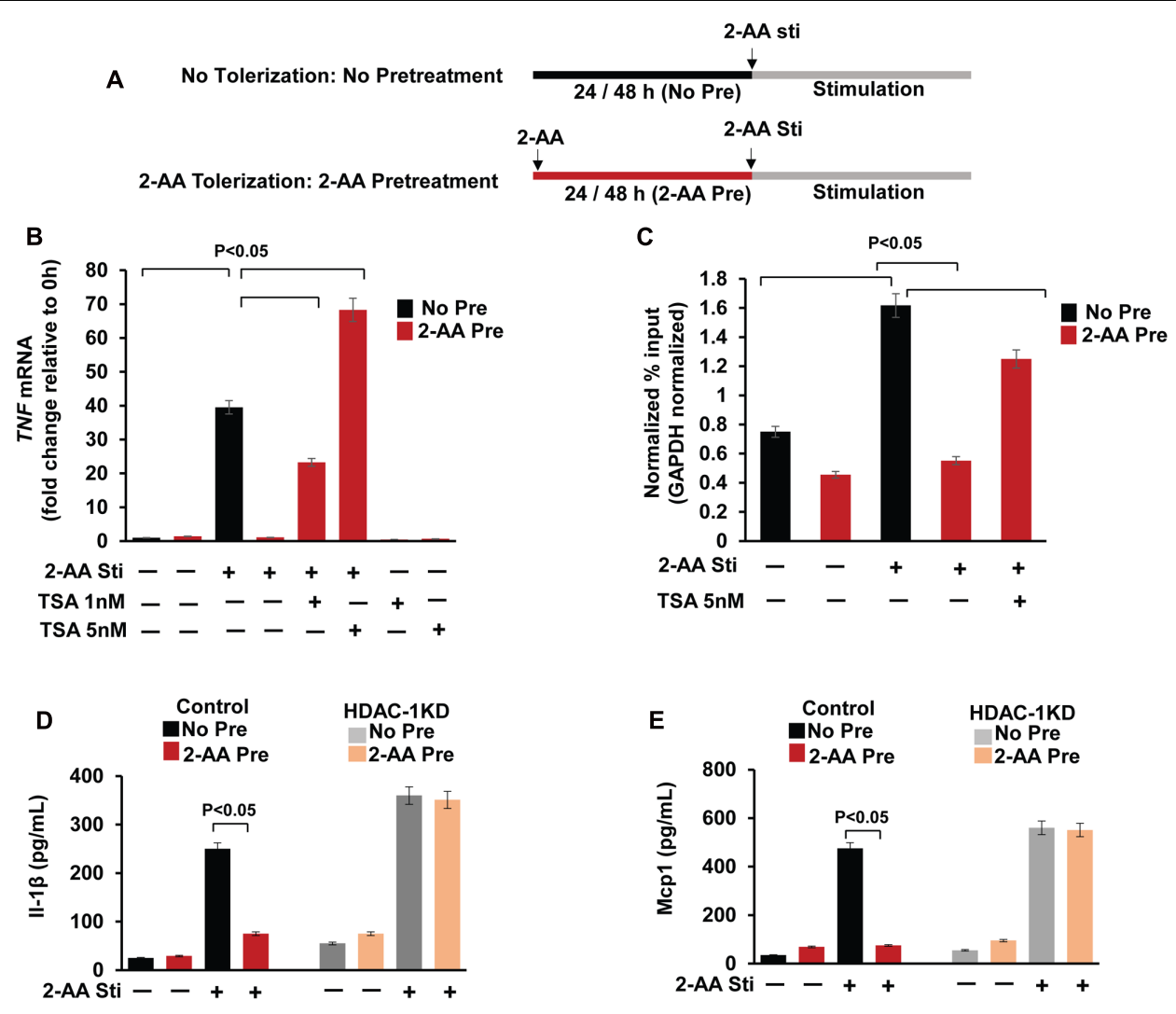

FIGURE 2 | Pharmacological and genetic inhibition of HDAC1 rescue the 2-AA-mediated immunomodulation. (A) Schematic representation of the 2-AA treatments. The human monocytes THP-1 cells and mouse macrophage RAW264.7 cell cells were left untreated (No Pre: No Tolerization) or pretreated with 2-AA for 24 h or $48 \mathrm{~h}$ (2-AA Pre: 2-AA tolerization) respectively, and then stimulated (Sti) with 2-AA. (B) Expression of TNF (1 h) was measured in non-pretreated, 2-AA pretreated and 2-AA + TSA pretreated THP-1 cells following 2-AA stimulation. Transcript levels were assessed by qRT-PCR and normalized to GAPDH. (C) ChIP assay of H3K18ac at the TNF promoter of 2-AA-pretreated, 2-AA and TSA pretreated or non-pretreated THP-1 cells following $3 \mathrm{~h} 2$-AA stimulation, assessed by qRT-PCR with primer covering the promoter site region of TNF relative to GAPDH. (D,E) ELISA of IL-1 $\beta$ and Mcp1 secretion in culture supernatant of 2-AA pretreated vector control RAW264.7 and HDAC1 KD cells following 6 h 2-AA stimulation ( $n=3$; means \pm SDs; $p<0.05$, Student's $t$-test).

from each sample using the Bradford protein assays kit (BioRad Laboratories, Hercules, United States). Fifteen to twenty-five micrograms of total protein was added to $1 \times$ Laemmli buffer, boiled for $10 \mathrm{~min}$, separated by SDS $7.5-15 \%$ polyacrylamide gel electrophoresis (PAGE) in $25 \mathrm{mM}$ Tris/250 mM glycine/0.1\% SDS buffer (Bio-Rad), and transferred to PVDF membranes (Bio-Rad). The membranes were blocked for $2 \mathrm{~h}$ in $5 \%$ non-fat milk. The membranes were then washed three times in TBS-T (20 mM Tris-HCl/150 mM NaCl/0.1\% Tween 20) and probed overnight with antibodies specific for acetyl-CBP/p300K1499, acetyl-NF-кBp65K310, HDAC1 (Cell Signaling Technology), CBP, p300, p65, and p50 (Santa Cruz Biotechnology, United States) at a dilution of 1:1,000, and mouse anti- $\beta$-actin (Santa Cruz Biotechnology) at a dilution of 1:2,000. Following three washes in TBST, the membranes were incubated with secondary horseradish peroxidase (HRP)-conjugated antibody: goat anti-rabbit (Santa Cruz Biotechnology, United States) or goat anti-mouse secondary antibodies (Promega, United States). The membranes were washed three times in TBST and then developed by SuperSignal West Pico Chemiluminescent Substrate (Thermo Scientific, United States), as per the manufacturer's instructions. Protein bands were quantified by ImageJ software ${ }^{1}$.

\section{Preparation of Nuclear Fraction}

Nuclear fractions were extracted from cells with a Nuclear Extract kit (Active Motif, Carlsbad, United States) as per manufacturer's instructions. Briefly, cells were scraped into a hypotonic buffer supplemented with phosphatase inhibitors and kept on ice. The cell suspension was centrifuged at $12,000 \mathrm{rpm}$ for $15 \mathrm{~min}$ at $4^{\circ} \mathrm{C}$. The cell pellet was suspended in nuclear lysis buffer, rocked lightly for $30 \mathrm{~min}$ at $4^{\circ} \mathrm{C}$, and centrifuged at $12,000 \mathrm{rpm}$ for $15 \mathrm{~min}$ at $4^{\circ} \mathrm{C}$. Nuclear protein was quantified by Bradford protein assay.

\section{Measurement of HAT Activity}

HAT activity was performed by using a non-radioactive HAT activity assay kit (Active Motif, United States), as per manufacturer's instructions. Briefly, the nuclear protein $(3 \mu \mathrm{g})$ was incubated in HAT assay buffer containing mixture of acetyl$\mathrm{CoA}$ and histone $\mathrm{H} 3$ peptide for $30 \mathrm{~min}$ at RT. The reaction

\footnotetext{
${ }^{1}$ http://rsb.info.nih.gov/ij/index.html
} 
was terminated by stop solution. The sample was incubated with developing solution for $15 \mathrm{~min}$ at RT. Fluorescence (excitation, $380 \mathrm{~nm}$; emission, $460 \mathrm{~nm}$ ) was measured using a microplate reader (Tecan Group Ltd, Switzerland). HAT activity was expressed as arbitrary fluorescence units (AFU).

\section{Histone H3 Modification Array}

The binding of a 2-AA treated nuclear fraction was used to identify the substrates of histone modifying enzymes using PreSure Histone $\mathrm{H} 3$ peptide Array ELISA kit (EpiGentek) according to the manufactures protocol. Briefly, $50 \mu \mathrm{L}$ of 2-AA-treated nuclear fraction was added to the histone $\mathrm{H} 3 \mathrm{~K}$ acetylated peptide-coated well and incubated for $1 \mathrm{~h}$. Following three washes developer solution was added for $10 \mathrm{~min}$ at RT and the absorbance at $450 \mathrm{~nm}$ was determined using a Sunrise plate reader.

\section{Chromatin Immunoprecipitation}

Cells were cross-linked in 1\% methanol-free formaldehyde for $10 \mathrm{~min}$, then placed in $0.125 \mathrm{M}$ glycine for $5 \mathrm{~min}$ at RT. Using the truChIP ${ }^{\mathrm{TM}}$ High Cell Chromatin Shearing kit (Covaris, United States), cells were prepared for sonication according to the manufacturer's protocol. Approximately $1 \times 10^{7}$ cells were places in a $12 \mathrm{~mm} \times 12 \mathrm{~mm}$ tube and subjected to shearing with the Covaris S220 sonicator for $8 \mathrm{~min}$ (140 peak power, 5 duty factor, 200 cycles/burst). The Magna ChIP ${ }^{\mathrm{TM}}$ A/G Kit (Millipore, United States) was used for the subsequent immunoprecipitations (IPs) according to the manufacturer's protocol. Briefly, chromatin from approximately $10^{6}$ cells was incubated overnight at $4^{\circ} \mathrm{C}$ with $4 \mu \mathrm{g}$ of anti-acetyl H3K18 (Abcam, United States) chromatin IP (ChIP)-grade antibody and $20 \mu \mathrm{L}$ of A/G magnetic beads. The beads were washed serially ( $5 \mathrm{~min}$ each) with low-salt wash buffer, high-salt wash buffer, $\mathrm{LiCl}$ wash buffer, and TE buffer from the kit at $4^{\circ} \mathrm{C}$. Chromatin was eluted with elution buffer containing Proteinase $\mathrm{K}$ at $62^{\circ} \mathrm{C}$ for $4 \mathrm{~h}$, then incubated at $95^{\circ} \mathrm{C}$ for $10 \mathrm{~min}$. DNA was isolated by column purification. Real-time PCR was performed with the Brilliant II SYBR green super mix (Agilent, United States) and primer sets to amplify various regions of TNF (forward: CCCCCTCGGAATCGGA, reverse: GAGCTCATCTGGAGGAAGCG) and GAPDH (forward: CGGTGCGTGCCCAGTT, reverse: CCCTACTTTCTCCCCGC TTT) loci. Normalized values were calculated by the percentinput method relative to the GAPDH promoter locus. The assay was conducted in triplicate; means are reported with standard deviations.

\section{Co-immunoprecipitation}

For protein-protein interaction assays, whole cell lysates were extracted in $1 \times$ TNT buffer [20 mM Tris-HCl, pH 7.5/200 mM $\mathrm{NaCl} /$ Triton X-100/0.1 M phosphatase inhibitor cocktails 1 and 2 (ingredients from Sigma-Aldrich)]. A total of $100 \mu \mathrm{g}$ aliquots of whole cell extracts were subjected to IP in $0.1 \times$ TNT buffer. IP was performed with $2 \mu \mathrm{g}$ of HDAC1, p300, CBP, p50, or p65 (Santa Cruz Biotechnology, Santa Cruz, CA, United States) and $50 \mu \mathrm{L}$ of Pierce protein A/G agarose beads (Thermo Scientific, United States)at $4^{\circ} \mathrm{C}$ for $3 \mathrm{~h}$. After washing three times with $0.1 \times$ TNT buffer, the bound proteins eluted in the Laemmli loading buffer supplemented with $100 \mathrm{mM}$ dithiothreitol by boiling for $10 \mathrm{~min}$, the precipitated proteins were analyzed by Western blotting, as described above.

\section{Measurement of IL-1 $\beta$ and Mcp1 by Enzyme-Linked Immunosorbent Assay}

Murine interleukin (IL)-1 $\beta$ and monocyte chemotactic protein (Mcp)1 were measured by ELISA using the Quantikine kits (R\&D Systems, MN, United States), following manufacturer's instructions. Briefly, culture supernatants were added to antibody-coated ELISA plates and kept at RT for $2 \mathrm{~h}$. Following four washes, HRP-conjugated streptavidin/biotinylated antibody solution was added to plates and kept at RT for $2 \mathrm{~h}$. The assay was developed with the tetramethylbenzidine substrate reagent and incubated at RT for $20 \mathrm{~min}$. The absorbance was assessed at $450 \mathrm{~nm}$ using a Sunrise plate reader.

\section{MTT Assay for Cell Cytotoxicity}

The cytotoxicity of cells treated with C 646 (Abcam, United States) was measured by 3-(4,5-dimethyl-2-thiazolyl)-2,5diphenyl-2H-tetrazolium bromide (MTT) assay. MTT (Sigma) stock solution (5 mg/mL PBS) was further diluted 1:5 in PBS. Cells were exposed to $200 \mu \mathrm{L}$ of this working solution in 96-well culture plates where dissolved MTT was allowed to convert to insoluble purple formazan via mitochondrial activity for $2 \mathrm{~h}$ at $37^{\circ} \mathrm{C}$ in humidified incubator containing $5 \% \mathrm{CO}_{2}$. The medium was discarded, formazan was solubilized with 95\% isopropanol-5\% formic acid for $10 \mathrm{~min}$ and absorbance was measured at $555 \mathrm{~nm}$ (reference wavelength $690 \mathrm{~nm}$ ) using a Sunrise plate reader. The concentration of C646 used did not induce cytotoxicity (Supplementary Figure S1).

\section{Pharmacological Inhibitors}

For treatment, THP-1 cells were incubated with HDAC1 inhibitor TSA (trichostatin A; Villagra et al., 2010) (1 or 5 nM, SigmaAldrich) and CBP/p300 inhibitor C646 (0.1 $\mu \mathrm{M}$; Zhao et al., 2015).

\section{Statistical Analysis}

For the analysis of statistical significance, the data were analyzed using the Student's $t$-test and $P$-value $<0.05$ was considered significant for all experiment.

\section{RESULTS}

\section{HDAC1 Controls the Dampening of Pro-inflammatory Responses in 2-AA-Tolerized Cells}

HDACs mediate histone deacetylation and thus suppress gene transcription (Delcuve et al., 2012). Our previous study demonstrates that $\mathrm{HDAC} 1$ regulates the deacetylation of $\mathrm{H} 3 \mathrm{~K} 18$ at the TNF- $\alpha$ promoter and TNF secretion in 2-AA pretreated (tolerized) cells (Bandyopadhaya et al., 2016b; Figure 1). We interrogated reversion of the deacetylation effect promoted by 2-AA by assessing the TNF mRNA levels in 2-AA-tolerized 
THP-1 cells in the presence of Class I/II HDAC inhibitor TSA (Figure 2A). Indeed, Figure 2B shows that in presence of TSA there is a significant increase of TNF mRNA expression in 2-AA pretreated cells. We additionally analyzed $\mathrm{H} 3 \mathrm{~K} 18$ acetylation at the TNF promoter. Pretreatment with 2 -AA and TSA enriched H3K18 acetylation at the TNF promoter compared to 2-AAtolerized THP-1 cells (Figure 2C). Furthermore, Figures 2D,E show that the secretion of the other major pro-inflammatory cytokines IL-1 $\beta$ and Mcp1 is significantly dampened in 2-AAtolerized cells, whereas depletion of HDAC1 counteracts the IL-1 $\beta$ and Mcp1 secretion in 2-AA-tolerized cells.

\section{2-AA-Tolerized Cells Lack NF-кB p65 Acetylation}

Post-translational modifications, particularly acetylation of NF$\kappa \mathrm{B}$ p65 enhances the DNA-binding activity of $\mathrm{p} 65$ at the promoter of pro-inflammatory cytokines regulated by NF- $\kappa \mathrm{B}$ (Chen et al., 2005; Bhatt and Ghosh, 2014). Our prior study demonstrated that 2-AA tolerization dampens the DNA-binding activity of p65 (Bandyopadhaya et al., 2012; Figure 1). Therefore, we sought to determine changes in the level of p65 acetylation following 2-AA treatment by using wild type and knockdown HDAC1 RAW264.7 cells. Figure 3 shows that although p65 acetylation is promoted following 2-AA stimulation, it is absent in 2-AA-tolerized cells (Figures 1, 3A). On the other hand, knockdown of HDAC1 restores p65 acetylation in 2-AA-tolerized cells (Figure 3B).

\section{2-AA Induces H3K18 Acetylation by CBP/p300}

Previously, we have found that 2-AA induces HAT activity and this induction was reduced in 2-AA-tolerized cells (Bandyopadhaya et al., 2016b). Our present data demonstrate that 2-AA-mediated HAT activity is regulated specifically by $\mathrm{CBP} / \mathrm{p} 300$, since C646 (the specific inhibitor of $\mathrm{p} 300 / \mathrm{CBP}$ HAT activity) abrogated nuclear HAT activity in THP-1 cells stimulated with 2-AA (Figure 4A). 2-AA initially stimulates the acetylation of $\mathrm{CBP} / \mathrm{p} 300$ at Lys1499, whereas 2-AA tolerization significantly dampens this acetylation mark in both THP-1 and
RAW264.7 cells (Figure 4B and Supplementary Figures S1, S2). Therefore, we used the 2-AA stimulated nuclear fraction to screen various residues for $\mathrm{H} 3 \mathrm{~K}$ acetylation. Figure 5 shows that 2-AA stimulated nuclear fractions have strong affinity toward K3K18 acetylated peptide, demonstrating that 2-AA modulates $\mathrm{H} 3 \mathrm{~K} 18$ acetylation, presumably by regulating the acetylation of $\mathrm{CBP} / \mathrm{p} 300$ (Figure 5).

\section{2-AA Enhances the Interaction between p50 and HDAC1 by Inhibiting p65 Phosphorylation and Disrupting p65 and CBP/p300 Interaction}

$\mathrm{NF}-\kappa \mathrm{B}$ signaling enhances the interaction between the active p65-p50 heterodimer and $\mathrm{CBP} / \mathrm{p} 300$, which displaces the silencing p50-p50 homodimer-HDAC1 complex from DNA (Zhong et al., 2002). We therefore investigated the proteinprotein interactions mediated by 2-AA and observed that the upregulation of HDAC1 protein expression in 2-AAtolerized cells (Bandyopadhaya et al., 2016b) was accompanied by enhanced interaction with p50 in THP-1 and RAW264.7 cell lines (Figure 6A and Supplementary Figure 3a) and disrupted interaction between p65 and CBP/p300 (Figure 6B and Supplementary Figure $3 \mathbf{b}$ ). 2-AA cell tolerization prior to stimulation resulted in reduced $\mathrm{CBP} / \mathrm{p} 300$ acetylation (Figure 4B and Supplementary Figure S2), a modification that dictates its binding partner (Zhong et al., 2002). Acetylation of lys310 of the p65 subunit enhances CBP/p300 HAT binding and disrupts HDAC1 binding to regulate transcriptional activity (Quivy and Van Lint, 2004) and this post-translational modification was also decreased in 2-AA-tolerized RAW264.7 cells, compared to non-tolerized cells that were stimulated only (Figure 6B). Importantly, Figure 6C shows that knockdown of $\mathrm{HDAC1}$ restored p300 and p65 interactions in 2-AA-tolerized and reexposed RAW264.7 cells that were otherwise disrupted in similar control cells. This shift in protein-protein interaction and HDAC1 regulation likely mediates changes in histone acetylation by 2 -AA resulting in the attenuation of pro-inflammatory cytokine expression.
A

\section{Control}

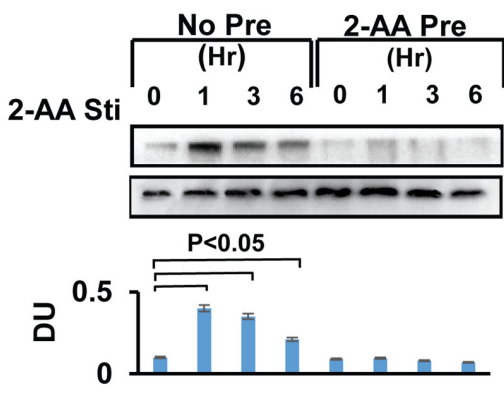

B

HDAC1 KD

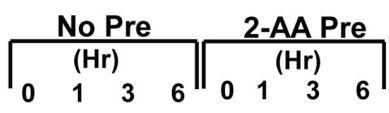

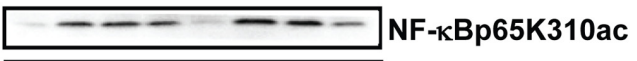

$\rightarrow-\infty-\infty-\infty-\infty \mathrm{NF}-\mathrm{kP} 65$

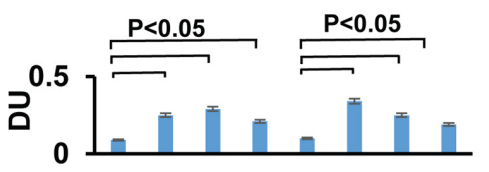

FIGURE 3 | 2-AA tolerization decreases the acetylation of NF-kBp65. Immunoblot showing decreased NF-kBp65K310 acetylation in 2-AA-tolerized RAW264.7 cells (A). HDAC1 KD restored NF-kBp65K310 acetylation in 2-AA-tolerized cells following 2-AA stimulation (B). Data are representative of three independent experiments. An asterisk $\left(^{*}\right)$ indicates significant difference from untreated cells $(P<0.05)$. DU, densitometry units, bars represent SD. 


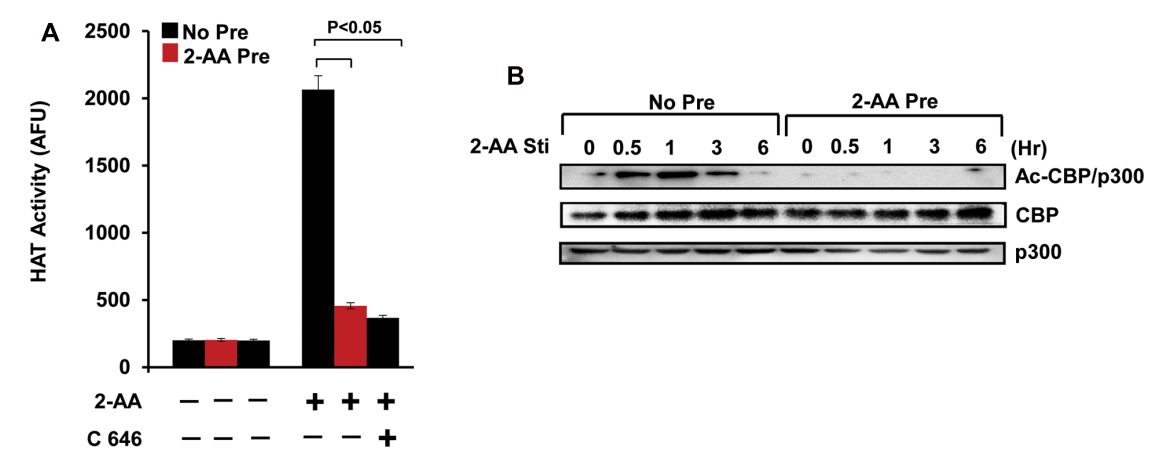

FIGURE 4 | 2-AA modulates CBP/p300-mediated HAT activity and the acetylation of CBP/p300. (A) HAT activity in nuclear lysate of 2-AA stimulated and 2-AA-tolerized THP-1 cells following $1 \mathrm{~h} 2$-AA stimulation $+/$ - of C646 ( $n=3$; means \pm SDs; $p<0.05$, Student's $t$-test). (B) Immunoblot showing decreased $\mathrm{CBP} / \mathrm{p} 300$ acetylation in 2-AA-tolerized THP-1 cells. Data are representative of three independent experiments.

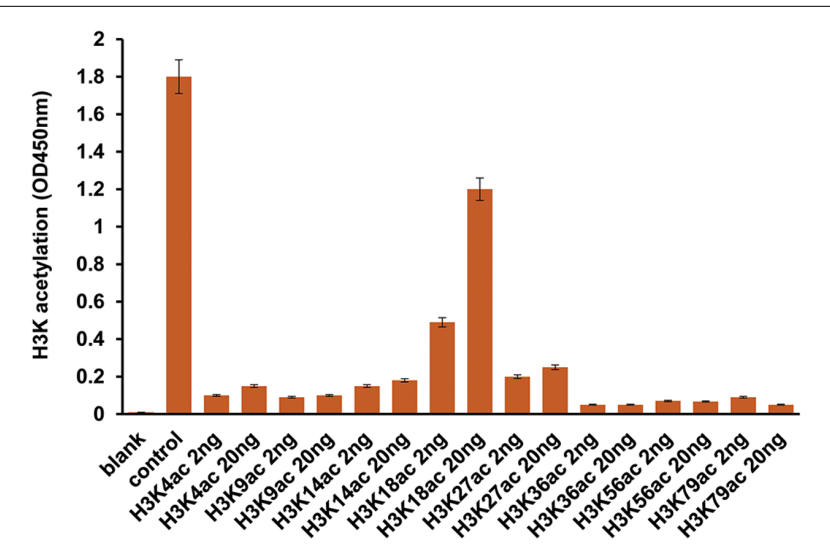

FIGURE 5 | 2-AA modulates H3K18 acetylation. Screening for Histone H3 acetylation following 2-AA stimulation. Nuclear extraction was prepared from THP-1 cells $1 \mathrm{~h}$ post-stimulation with 2-AA. The interaction of the enzyme in the sample solution with the acetylated $\mathrm{H} 3 \mathrm{~K}$ peptides was measured by using Histone H3 Peptide Array ELISA kit (number of replicates = 2; means \pm SDs).

\section{DISCUSSION}

Pathogens may modulate host immune responses in a way to avoid clearance and potentially favor their long-term presence in the host tissues (Ruby and Monack, 2011; Reddick and Alto, 2014; Ribet and Cossart, 2015). Recently, we have demonstrated that the bacterial excreted QS small molecule 2-AA trains the host to tolerate a sustained presence of $P$. aeruginosa by a HDAC1-mediated epigenetic reprogramming (Bandyopadhaya et al., 2016b). In this study, we undertook further molecular analyses of alterations in intracellular signaling components and epigenetic regulators following 2-AA-mediated tolerization. Our results reveal that the 2-AA promoted host tolerance implicates NF-кBp50 and HDAC1 interaction. Several data are in support of this conclusion, including that: (i) 2-AA-tolerized cells lack NF-кBp65 acetylation; (ii) 2-AA modulates H3K18 acetylation via downregulation of $\mathrm{CBP} / \mathrm{p} 300 \mathrm{HAT}$ activity and expression; (iii) 2-AA tolerization induces enhanced proteinprotein interaction between NF-кBp50 and HDAC1 and reduces binding of $\mathrm{CBP} / \mathrm{p} 300$ to $\mathrm{NF}-\kappa \mathrm{B}$ p65; (iv) HDAC1 knockdown restores acetylation of NF- $\mathrm{B}$ p65 in 2-AA-tolerized cells; and (v) the pharmacological and genetic inhibition of HDAC1 rescue 2-AA-mediated immunomodulation observed. These findings provide additional details on the components and mechanism involved in the epigenetic reprogramming reported previously (Bandyopadhaya et al., 2016b) and strongly suggest that 2AA tolerization triggers modulation of intracellular molecules that contributes to the shifts in innate immune cells from transcriptionally permissive to a repressive chromatin state that in turn augments host tolerance to pathogen's long-term presence we reported previously (Bandyopadhaya et al., 2012, 2016b).

Previous studies have demonstrated that diminished NF$\kappa \mathrm{B}$ signaling (Medvedev et al., 2000; Dobrovolskaia et al., 2003; Bandyopadhaya et al., 2016b), p50-p50 homodimers (Ziegler-Heitbrock et al., 1994; Bandyopadhaya et al., 2012), chromatin remodeling (Bandyopadhaya et al., 2016b), and dampened inflammatory response (Biswas and Lopez-Collazo, 2009; Bandyopadhaya et al., 2012) have all been implicated in the induction of the tolerant state. NF- $\kappa$ B p50-HDAC1 complex represses transcription of major proinflammatory mediators, such as, Tnf, IL-6, and cyclooxygenase-2 (Cox-2) (Oakley et al., 2005; Elsharkawy et al., 2010). Our data showed that HDAC1 is associated with deacetylation of $\mathrm{H} 3 \mathrm{~K} 18$ at $\operatorname{Tnf}$ promoters in the 2-AA-tolerized cells (Bandyopadhaya et al., 2016b). Here we provide evidence suggesting that inhibition of HDAC1 activity results in an increase of pro-inflammatory mediators in 2-AAtolerized cells. We speculate that the increased interaction of p50 and HDAC1 in 2-AA-tolerized cells may orchestrate the transcriptional repression of pro-inflammatory genes. NF- $\kappa B$ p50-HDAC3-nuclear hormone receptor co-repressor interaction was found to be required to repress inflammatory activation in LPS-tolerized cells (Yan et al., 2012) that is different from the 2-AA-mediated tolerization (Bandyopadhaya et al., 2016b). Thus, HDAC1 and HDAC3 appear to be critically involved in immunomodulation by epigenetic reprogramming mediated by bacterial products, while HDAC1 also regulates the ability to tolerate pathogen burden (Bandyopadhaya et al., 2016b). 


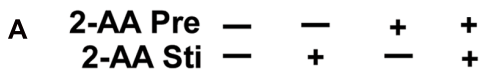 \\ \begin{tabular}{lll}
\hline 1 & 1 & HB: HDAC1
\end{tabular} \\ IP: p50

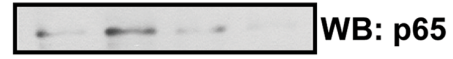 \\ WB: $p 50$ \\ IP: HDAC1

$-\infty$ WB: p50
- WB: HDAC1

B

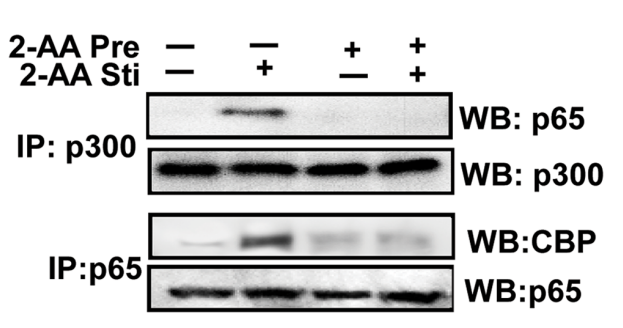

C

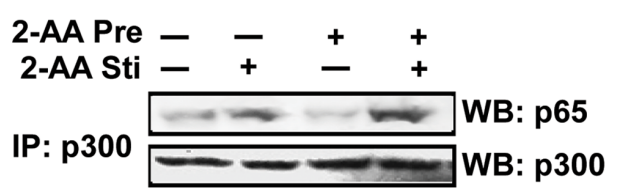

FIGURE 6 | 2-AA tolerization induces interaction between NF-кB p50 and HDAC1 and disrupts p65-CBP/p300 binding. (A) Co-IP assay showing p50/HDAC1 interaction in 2-AA stimulated and 2-AA-tolerized THP-1 cells following at $1 \mathrm{~h} 2-\mathrm{AA}$ stimulation (top blot, far right band). (B,C). Co-IP assay confirming that 2-AA tolerization inhibits the interaction of p65 with CBP and p300 in vector control RAW264.7 cells (B), and demonstrating an increased interaction of p300-p65 in HDAC1 KD cells (C) following $1 \mathrm{~h}$ 2-AA stimulation (1 h). WB, Western blot; Co-IP, co-immunoprecipitation. Data are representative of three independent experiments.

HDAC1 and NF- $\kappa \mathrm{B}$ signaling coordinate to repress inflammatory genes during infection (Zhou et al., 2013). NF- $\kappa \mathrm{B}$ influences transcription by recruiting p300/CBP, which can specifically acetylate the H3K18 residue (Jin et al., 2011) and simultaneously regulate p65 acetylation, leading to transcription (Quivy and Van Lint, 2004). It has been reported that of NF-кB acetylation is required for NF- $\kappa \mathrm{B}$-dependent transcription (Chen et al., 2005) and is regulated by the HDAC family of proteins, including HDAC1 (Ashburner et al., 2001). Here our results show that 2-AA tolerization promotes the interaction between p50 and HDAC1, with a coordinate loss of p65 (Lysine 310) acetylation and $\mathrm{CBP} / \mathrm{p} 300$ binding with p65, which leads to a transcriptionally repressive state. It has been reported that Sirtuin 1 (SIRT1, Class III HDAC) deacetylates p65 at Lysine 310 to repress the pro-inflammatory responses during initiation of endotoxin tolerance (Liu et al., 2011). This study provides further demonstration of the intimate link between HDACs and NF- $\kappa$ B pathway, specifically between HDAC1 and components of the $\mathrm{NF}-\kappa \mathrm{B}$ signaling cascade in 2-AA-mediated host tolerance.

Based on our published and present results, we propose a model in Figure 1, in which 2-AA tolerization, which involves pretreatment and stimulation (Figure 2A), decreases $\mathrm{CBP} / \mathrm{p} 300$ HAT activity, inhibits p65 and H3 acetylation, prevents $\mathrm{p} 65$ and $\mathrm{CBP} / \mathrm{p} 300$ interaction, and in turn promotes p50-HDAC1 interaction, leading to a sustained 2-AAmediated histone deacetylation, concomitant loss of active transcription, immunosuppression and long-term bacterial presence (Bandyopadhaya et al., 2012, 2016b). It appears that host tolerance training by 2-AA represses the inflammatory response via disruption of $\mathrm{p} 65: \mathrm{CBP} / \mathrm{p} 300$ (active) complex and induction of p50:HDAC1 (inactive) complex. Further studies are in progress to address this regulatory mechanisms.

Consistent with our model, several studies reported that increased levels of $\mathrm{HDAC1}$ and reduction of histone $\mathrm{H} 3$ acetylation allows persistent Mycobacterium tuberculosis infection (Chandran et al., 2015), and promotes long-term survival of Anaplasma phagocytophilum in immune cells (Rennoll-Bankert and Dumler, 2012). Interestingly, it has been also reported that NF- $\mathrm{B}$ p50 promotes latency of the human immunodeficiency virus involving HDAC1 recruitment and transcriptional repression (Williams et al., 2006). Our present study describes an intimate connection between histone acetylation/deacetylation status and relevant molecular mechanisms of intracellular signaling components. This connection most likely coordinates the 2-AA-mediated host tolerance to infection.

Collectively, our results strongly indicate that 2-AA in tolerized cells acts as a switch to maintain the chromatin state from a transcriptionally active to a "silent" state, modulating the host inflammatory signaling cascade epigenetically and involving protein-protein interactions between histone acetylase/deacetylases and p50/p65 NF-кB subunits. Taken 
together, we conclude that 2-AA-mediated host tolerance to bacterial burden is a result of a coordinated modulation of intracellular signaling and epigenetics regulators that leads to long-lasting epigenetic alterations. This coordinated effort may contribute to the long-term bacterial presence and host resilience to bacterial burden we observed to occur in vivo in tolerized animals (Bandyopadhaya et al., 2012, 2016b). The data presented here provide further evidence that QS signals play an important regulatory role in modulating host intracellular signaling pathways, and uncover aspects of immunoregulation that may contribute to the HT/R promoted by the QS molecule 2-AA. This interplay between 2-AA and host can lead to a co-evolutionary arms race, where the pathogen and host are continuously selected to avoid the cost of infection and the cost of immune clearance. Given that QS is a conserved mechanism of prokaryotes, it is likely that 2-AA-like molecules that promote similar effects may exist in other pathogens. Overall, the results presented promote a better understanding of the biological mechanisms mediating mutual pathogen-host adaptation and the causes and consequences of variation in HT/R that remain extremely limited.

\section{AUTHOR CONTRIBUTIONS}

$\mathrm{AB}$ and $\mathrm{LR}$ conceived and designed the study. $\mathrm{AB}$ and LR wrote the manuscript. $\mathrm{AB}$ and $\mathrm{AT}$ performed experiments and data analysis.

\section{REFERENCES}

Ashburner, B. P., Westerheide, S. D., and Baldwin, A. S. Jr. (2001). The p65 (RelA) subunit of NF-kappaB interacts with the histone deacetylase (HDAC) corepressors HDAC1 and HDAC2 to negatively regulate gene expression. Mol. Cell. Biol. 21, 7065-7077. doi: 10.1128/MCB.21.20.7065-7077.2001

Ayres, J. S., and Schneider, D. S. (2012). Tolerance of infections. Annu. Rev. Immunol. 30, 271-294. doi: 10.1146/annurev-immunol-020711-075030

Bandyopadhaya, A., Constantinou, C., Psychogios, N., Ueki, R., Yasuhara, S., Martyn, J. A., et al. (2016a). Bacterial-excreted small volatile molecule 2aminoacetophenone induces oxidative stress and apoptosis in murine skeletal muscle. Int. J. Mol. Med. 37, 867-878. doi: 10.3892/ijmm.2016.2487

Bandyopadhaya, A., Kesarwani, M., Que, Y. A., He, J., Padfield, K., Tompkins, R., et al. (2012). The quorum sensing volatile molecule 2 -amino acetophenon modulates host immune responses in a manner that promotes life with unwanted guests. PLoS Pathog. 8:e1003024. doi: 10.1371/journal.ppat.1003024

Bandyopadhaya, A., Tsurumi, A., Maura, D., Jeffrey, K. L., and Rahme, L. G. (2016b). A quorum-sensing signal promotes host tolerance training through HDAC1-mediated epigenetic reprogramming. Nat. Microbiol. 1:16174. doi: 10.1038/nmicrobiol.2016.174

Bhatt, D., and Ghosh, S. (2014). Regulation of the NF-kappaB-mediated transcription of inflammatory genes. Front. Immunol. 5:71. doi: 10.3389/fimmu. 2014.00071

Bierne, H., Hamon, M., and Cossart, P. (2012). Epigenetics and bacterial infections. Cold Spring Harb. Perspect. Med. 2:a010272. doi: 10.1101/cshperspect.a010272

Biswas, S. K., and Lopez-Collazo, E. (2009). Endotoxin tolerance: new mechanisms, molecules and clinical significance. Trends Immunol. 30, 475-487. doi: 10.1016/ j.it.2009.07.009

Chandran, A., Antony, C., Jose, L., Mundayoor, S., Natarajan, K., and Kumar, R. A. (2015). Mycobacterium tuberculosis infection induces HDAC1-mediated

\section{FUNDING}

This work was supported by Shriners grants (nos. 87100 and 85200) and in part by NIH R33AI105902 and Cystic Fibrosis Foundation grant no. CFF\#11P0. The funders had no role in study design, data collection and analysis, decision to publish or preparation of the manuscript. AT was supported by the Shriners Hospitals Research Fellowship \#84293.

\section{SUPPLEMENTARY MATERIAL}

The Supplementary Material for this article can be found online at: http://journal.frontiersin.org/article/10.3389/fmicb. 2017.01211/full\#supplementary-material

FIGURE S1 | Human monocyte cell viability in presence of HAT inhibitor C646. MTT assay measuring viability in THP-1 cells following treatment with increasing concentrations of C646. Cells were exposed to C646 for $24 \mathrm{~h}(n=3$; means \pm SDs).

FIGURE S2 | Acetylation of CBP/p300 is modulated by 2-AA. Western blot analysis showing decreased CBP/p300 acetylation in 2-AA-tolerized mouse macrophage cells RAW264.7 cells following 2-AA stimulation (1 h). Data are representative of three independent experiments.

FIGURE S3 | 2-AA tolerization induces interaction between NF-kB p50 and HDAC1 and disrupts p65-CBP/p300 binding. (a) Co-IP assay showing increased p50/HDAC1 interaction in 2-AA-tolerized mouse macrophage RAW264.7 cells following 2-AA stimulation (1 h). (b) Co-IP assay confirming that 2-AA tolerization inhibits interaction of $\mathrm{p} 65$ with CBP and $\mathrm{p} 300$ in THP-1 cells. WB, Western blot. Data are representative of three independent experiments.

suppression of IL-12B gene expression in macrophages. Front. Cell Infect. Microbiol. 5:90. doi: 10.3389/fcimb.2015.00090

Chen, L. F., Williams, S. A., Mu, Y., Nakano, H., Duerr, J. M., Buckbinder, L., et al. (2005). NF-kappaB RelA phosphorylation regulates RelA acetylation. Mol. Cell. Biol. 25, 7966-7975. doi: 10.1128/MCB.25.18.7966-7975.2005

Cox, C. D., and Parker, J. (1979). Use of 2-aminoacetophenone production in identification of Pseudomonas aeruginosa. J. Clin. Microbiol. 9, 479-484.

Delcuve, G. P., Khan, D. H., and Davie, J. R. (2012). Roles of histone deacetylases in epigenetic regulation: emerging paradigms from studies with inhibitors. Clin. Epigenet. 4:5. doi: 10.1186/1868-7083-4-5

Deziel, E., Gopalan, S., Tampakaki, A. P., Lepine, F., Padfield, K. E., Saucier, M., et al. (2005). The contribution of MvfR to Pseudomonas aeruginosa pathogenesis and quorum sensing circuitry regulation: multiple quorum sensing-regulated genes are modulated without affecting lasRI, rhlRI or the production of N-acyl-L-homoserine lactones. Mol. Microbiol. 55, 998-1014. doi: 10.1111/j.1365-2958.2004.04448.x

Diggle, S. P., Cornelis, P., Williams, P., and Camara, M. (2006). 4-quinolone signalling in Pseudomonas aeruginosa: old molecules, new perspectives. Int. J. Med. Microbiol. 296, 83-91. doi: 10.1016/j.ijmm.2006.01.038

Dobrovolskaia, M. A., Medvedev, A. E., Thomas, K. E., Cuesta, N., Toshchakov, V., Ren, T., et al. (2003). Induction of in vitro reprogramming by Toll-like receptor (TLR) 2 and TLR4 agonists in murine macrophages: effects of TLR "homotolerance" versus "heterotolerance" on NF-kappa B signaling pathway components. J. Immunol. 170, 508-519. doi: 10.4049/jimmunol.170.1.508

Drees, S. L., and Fetzner, S. (2015). PqsE of Pseudomonas aeruginosa acts as pathway-specific thioesterase in the biosynthesis of alkylquinolone signaling molecules. Chem. Biol. 22, 611-618. doi: 10.1016/j.chembiol.2015.04.012

Drees, S. L., Li, C., Prasetya, F., Saleem, M., Dreveny, I., Williams, P., et al. (2016). PqsBC, a condensing enzyme in the biosynthesis of the Pseudomonas aeruginosa quinolone signal: CRYSTAL STRUCTURE, INHIBITION, AND 
REACTION MECHANISM. J. Biol. Chem. 291, 6610-6624. doi: 10.1074/jbc. M115.708453

Elsharkawy, A. M., Oakley, F., Lin, F., Packham, G., Mann, D. A., and Mann, J. (2010). The NF-kappaB p50:p50:HDAC-1 repressor complex orchestrates transcriptional inhibition of multiple pro-inflammatory genes. J. Hepatol. 53, 519-527. doi: 10.1016/j.jhep.2010.03.025

Gellatly, S. L., and Hancock, R. E. (2013). Pseudomonas aeruginosa: new insights into pathogenesis and host defenses. Pathog. Dis. 67, 159-173. doi: 10.1111/ 2049-632X.12033

Gilmore, T. D. (2006). Introduction to NF-kappaB: players, pathways, perspectives. Oncogene 25, 6680-6684. doi: 10.1038/sj.onc.1209954

Hamon, M. A., and Cossart, P. (2008). Histone modifications and chromatin remodeling during bacterial infections. Cell Host Microbe 4, 100-109. doi: 10.1016/j.chom.2008.07.009

Holm, A., and Vikstrom, E. (2014). Quorum sensing communication between bacteria and human cells: signals, targets, and functions. Front. Plant Sci. 5:309. doi: 10.3389/fpls.2014.00309

Iwasaki, A., and Medzhitov, R. (2004). Toll-like receptor control of the adaptive immune responses. Nat. Immunol. 5, 987-995. doi: 10.1038/ni1112

Jimenez, P. N., Koch, G., Thompson, J. A., Xavier, K. B., Cool, R. H., and Quax, W. J. (2012). The multiple signaling systems regulating virulence in Pseudomonas aeruginosa. Microbiol. Mol. Biol. Rev. 76, 46-65. doi: 10.1128/MMBR.05007-11

Jin, Q., Yu, L. R., Wang, L., Zhang, Z., Kasper, L. H., Lee, J. E., et al. (2011). Distinct roles of GCN5/PCAF-mediated H3K9ac and CBP/p300-mediated H3K18/27ac in nuclear receptor transactivation. EMBO J. 30, 249-262. doi: 10.1038/emboj. 2010.318

Kerr, K. G., and Snelling, A. M. (2009). Pseudomonas aeruginosa: a formidable and ever-present adversary. J. Hosp. Infect. 73, 338-344. doi: 10.1016/j.jhin.2009. 04.020

Kesarwani, M., Hazan, R., He, J., Que, Y. A., Apidianakis, Y., Lesic, B., et al. (2011). A quorum sensing regulated small volatile molecule reduces acute virulence and promotes chronic infection phenotypes. PLoS Pathog. 7:e1002192. doi: 10.1371/journal.ppat.1002192

Kravchenko, V. V., Kaufmann, G. F., Mathison, J. C., Scott, D. A., Katz, A. Z., Grauer, D. C., et al. (2008). Modulation of gene expression via disruption of NF-kappaB signaling by a bacterial small molecule. Science 321, 259-263. doi: 10.1126/science.1156499

Liu, T. F., Yoza, B. K., El Gazzar, M., Vachharajani, V. T., and McCall, C. E. (2011). $\mathrm{NAD}+$-dependent SIRT1 deacetylase participates in epigenetic reprogramming during endotoxin tolerance. J. Biol. Chem. 286, 9856-9864. doi: 10.1074/jbc. M110.196790

Maura, D., Hazan, R., Kitao, T., Ballok, A. E., and Rahme, L. G. (2016). Evidence for direct control of virulence and defense gene circuits by the Pseudomonas aeruginosa quorum sensing regulator, MvfR. Sci. Rep. 6:34083. doi: 10.1038/ srep34083

Medvedev, A. E., Kopydlowski, K. M., and Vogel, S. N. (2000). Inhibition of lipopolysaccharide-induced signal transduction in endotoxin-tolerized mouse macrophages: dysregulation of cytokine, chemokine, and toll-like receptor 2 and 4 gene expression. J. Immunol. 164, 5564-5574. doi: 10.4049/jimmunol.164. 11.5564

Medzhitov, R., Schneider, D. S., and Soares, M. P. (2012). Disease tolerance as a defense strategy. Science 335, 936-941. doi: 10.1126/science.1214935

Meunier, I., Kaufmann, E., Downey, J., and Divangahi, M. (2017). Unravelling the networks dictating host resistance versus tolerance during pulmonary infections. Cell Tissue Res. 367, 525-536. doi: 10.1007/s00441-017-2572-5

Mogensen, T. H. (2009). Pathogen recognition and inflammatory signaling in innate immune defenses. Clin. Microbiol. Rev. 22, 240-273. doi: 10.1128/CMR. 00046-08

Newton, K., and Dixit, V. M. (2012). Signaling in innate immunity and inflammation. Cold Spring Harb. Perspect. Biol. 4:a006049. doi: 10.1101/ cshperspect.a006049

Ng, W. L., and Bassler, B. L. (2009). Bacterial quorum-sensing network architectures. Annu. Rev. Genet. 43, 197-222. doi: 10.1146/annurev-genet102108-134304

Oakley, F., Mann, J., Nailard, S., Smart, D. E., Mungalsingh, N., Constandinou, C., et al. (2005). Nuclear factor-kappaB1 (p50) limits the inflammatory and fibrogenic responses to chronic injury. Am. J. Pathol. 166, 695-708. doi: 10.1016/S0002-9440(10)62291-2
Parker, C. T., and Sperandio, V. (2009). Cell-to-cell signalling during pathogenesis. Cell Microbiol. 11, 363-369. doi: 10.1111/j.1462-5822.2008.01272.x

Que, Y. A., Hazan, R., Strobel, B., Maura, D., He, J., Kesarwani, M., et al. (2013). A quorum sensing small volatile molecule promotes antibiotic tolerance in bacteria. PLoS ONE 8:e80140. doi: 10.1371/journal.pone.0080140

Quivy, V., and Van Lint, C. (2004). Regulation at multiple levels of NF-kappaBmediated transactivation by protein acetylation. Biochem. Pharmacol. 68, 1221-1229. doi: 10.1016/j.bcp.2004.05.039

Raberg, L. (2014). How to live with the enemy: understanding tolerance to parasites. PLoS Biol. 12:e1001989. doi: 10.1371/journal.pbio.1001989

Reddick, L. E., and Alto, N. M. (2014). Bacteria fighting back: how pathogens target and subvert the host innate immune system. Mol. Cell. 54, 321-328. doi: 10.1016/j.molcel.2014.03.010

Rennoll-Bankert, K. E., and Dumler, J. S. (2012). Lessons from Anaplasma phagocytophilum: chromatin remodeling by bacterial effectors. Infect. Disord. Drug Targets 12, 380-387. doi: 10.2174/187152612804142242

Ribet, D., and Cossart, P. (2015). How bacterial pathogens colonize their hosts and invade deeper tissues. Microbes Infect. 17, 173-183. doi: 10.1016/j.micinf.2015. 01.004

Richardson, L. A. (2016). Understanding disease tolerance and resilience. PLoS Biol. 14:e1002513. doi: 10.1371/journal.pbio.1002513

Ruby, T., and Monack, D. M. (2011). At home with hostility: How do pathogenic bacteria evade mammalian immune surveillance to establish persistent infection? F1000 Biol. Rep. 3:1. doi: 10.3410/B3-1

Rumbaugh, K. P., and Kaufmann, G. F. (2012). Exploitation of host signaling pathways by microbial quorum sensing signals. Curr. Opin. Microbiol. 15, 162-168. doi: 10.1016/j.mib.2011.12.003

Rutherford, S. T., and Bassler, B. L. (2012). Bacterial quorum sensing: its role in virulence and possibilities for its control. Cold Spring Harb. Perspect. Med. 2:a012427. doi: 10.1101/cshperspect.a012427

Schmid-Hempel, P. (2009). Immune defence, parasite evasion strategies and their relevance for 'macroscopic phenomena' such as virulence. Philos. Trans. R. Soc. Lond. B Biol. Sci. 364, 85-98. doi: 10.1098/rstb.2008.0157

Scott-Thomas, A. J., Syhre, M., Pattemore, P. K., Epton, M., Laing, R., Pearson, J., et al. (2010). 2-Aminoacetophenone as a potential breath biomarker for Pseudomonas aeruginosa in the cystic fibrosis lung. BMC Pulm. Med. 10:56. doi: 10.1186/1471-2466-10-56

Sharma, V., Jordan, J. J., Ciribilli, Y., Resnick, M. A., Bisio, A., and Inga, A. (2015). Quantitative analysis of NF-kappaB transactivation specificity using a yeast-based functional assay. PLOS ONE 10:e0130170. doi: 10.1371/journal. pone. 0130170

Soares, M. P., Gozzelino, R., and Weis, S. (2014). Tissue damage control in disease tolerance. Trends Immunol. 35, 483-494. doi: 10.1016/j.it.2014.08.001

Soares, M. P., Teixeira, L., and Moita, L. F. (2017). Disease tolerance and immunity in host protection against infection. Nat. Rev. Immunol. 17, 83-96. doi: 10.1038/ nri.2016.136

Starkey, M., Lepine, F., Maura, D., Bandyopadhaya, A., Lesic, B., He, J., et al. (2014). Identification of anti-virulence compounds that disrupt quorumsensing regulated acute and persistent pathogenicity. PLoS Pathog. 10:e1004321. doi: 10.1371/journal.ppat.1004321

Tzika, A. A., Constantinou, C., Bandyopadhaya, A., Psychogios, N., Lee, S., Mindrinos, M., et al. (2013). A small volatile bacterial molecule triggers mitochondrial dysfunction in murine skeletal muscle. PLoS ONE 8:e74528. doi: 10.1371/journal.pone.0074528

Villagra, A., Sotomayor, E. M., and Seto, E. (2010). Histone deacetylases and the immunological network: implications in cancer and inflammation. Oncogene 29, 157-173. doi: 10.1038/onc.2009.334

Vo, N., and Goodman, R. H. (2001). CREB-binding protein and p300 in transcriptional regulation. J. Biol. Chem. 276, 13505-13508. doi: 10.1074/jbc. R000025200

Williams, P., and Camara, M. (2009). Quorum sensing and environmental adaptation in Pseudomonas aeruginosa: a tale of regulatory networks and multifunctional signal molecules. Curr. Opin. Microbiol. 12, 182-191. doi: 10.1016/j.mib.2009.01.005

Williams, S. A., Chen, L. F., Kwon, H., Ruiz-Jarabo, C. M., Verdin, E., and Greene, W. C. (2006). NF-kappaB p50 promotes HIV latency through HDAC recruitment and repression of transcriptional initiation. EMBO J. 25, 139-149. doi: 10.1038/sj.emboj.7600900 
Xiao, G., Deziel, E., He, J., Lepine, F., Lesic, B., Castonguay, M. H., et al. (2006). MvfR, a key Pseudomonas aeruginosa pathogenicity LTTR-class regulatory protein, has dual ligands. Mol. Microbiol. 62, 1689-1699. doi: 10.1111/j.13652958.2006.05462.x

Yan, Q., Carmody, R. J., Qu, Z., Ruan, Q., Jager, J., Mullican, S. E., et al. (2012). Nuclear factor-kappaB binding motifs specify Toll-like receptor-induced gene repression through an inducible repressosome. Proc. Natl. Acad. Sci. U.S.A. 109, 14140-14145. doi: 10.1073/pnas.1119842109

Yang, X. D., Tajkhorshid, E., and Chen, L. F. (2010). Functional interplay between acetylation and methylation of the RelA subunit of NF-kappaB. Mol. Cell. Biol. 30, 2170-2180. doi: 10.1128/MCB.01343-09

Yuan, H., Reddy, M. A., Sun, G., Lanting, L., Wang, M., Kato, M., et al. (2013). Involvement of $\mathrm{p} 300 / \mathrm{CBP}$ and epigenetic histone acetylation in TGF-beta1mediated gene transcription in mesangial cells. Am. J. Physiol. Renal Physiol. 304, F601-F613. doi: 10.1152/ajprenal.00523.2012

Zhao, D., Fukuyama, S., Sakai-Tagawa, Y., Takashita, E., Shoemaker, J. E., and Kawaoka, Y. (2015). C646, a novel p300/CREB-binding protein-specific inhibitor of histone acetyltransferase, attenuates influenza A virus infection. Antimicrob. Agents Chemother. 60, 1902-1906. doi: 10.1128/AAC.02055-15

Zhong, H., May, M. J., Jimi, E., and Ghosh, S. (2002). The phosphorylation status of nuclear NF-kappa B determines its association with $\mathrm{CBP} / \mathrm{p} 300$ or HDAC-1. Mol. Cell. 9, 625-636. doi: 10.1016/S1097-2765(02) 00477-X
Zhou, R., Gong, A. Y., Chen, D., Miller, R. E., Eischeid, A. N., and Chen, X. M. (2013). Histone deacetylases and NF-kB signaling coordinate expression of CX3CL1 in epithelial cells in response to microbial challenge by suppressing miR-424 and miR-503. PLoS ONE 8:e65153. doi: 10.1371/journal.pone.006 5153

Ziegler-Heitbrock, H. W., Wedel, A., Schraut, W., Strobel, M., Wendelgass, P., Sternsdorf, T., et al. (1994). Tolerance to lipopolysaccharide involves mobilization of nuclear factor kappa B with predominance of $\mathrm{p} 50$ homodimers. J. Biol. Chem. 269, 17001-17004.

Conflict of Interest Statement: LR is the scientific founder and scientific advisory board member of Spero Therapeutics LLC (no funding was received from Spero).

The other authors declare that the research was conducted in the absence of any commercial or financial relationships that could be construed as a potential conflict of interest.

Copyright (c) 2017 Bandyopadhaya, Tsurumi and Rahme. This is an open-access article distributed under the terms of the Creative Commons Attribution License (CC BY). The use, distribution or reproduction in other forums is permitted, provided the original author(s) or licensor are credited and that the original publication in this journal is cited, in accordance with accepted academic practice. No use, distribution or reproduction is permitted which does not comply with these terms. 